\title{
Corrigendum to Self-Synchronization of Populations of Nonlinear Oscillators in the Thermodynamic Limit ${ }^{1}$
}

\author{
L. L. Bonilla, ${ }^{2}$ J. M. Casado, ${ }^{2}$ and M. Morillo ${ }^{2}$
}

Received October 8, 1987

1. Due to a numerical instability, the true linear stability line for the stationary solution (2.3) differs near the $A$ axis from the dashed curve in Fig. 1. For small $\theta,(2.6 \mathrm{a})$ is approximately given by $A+1=\theta^{2}-\frac{1}{2} \theta^{4}+O\left(\theta^{6}\right)$. This implies that the dashed and solid lines in Fig. 1 should confound each other near the minimum $A=-1, \theta=0$. All phase transitions in the paper are thus second order.

2. The Liapunov functional for the time-periodic probability density $p_{0}(t, \mathbf{x})$ can be constructed by using Shiino's results. ${ }^{(1)}$ Instead of the relative entropy $H\left(p, p_{0}\right)$ as in Eq. (2.29), the Liapunov functional is $H(p, Q)$, where

$$
Q(t, \mathbf{x})=\exp \left\{-\phi(\mathbf{x})+J F^{-1}\left[2 \mathbf{x} \cdot\langle\mathbf{x}(t)\rangle-\langle\mathbf{x}(t)\rangle^{2}\right]\right\}
$$

Here $\langle\mathbf{x}(t)\rangle$ is the mean value corresponding to $p(t, \mathbf{x})$, the solution of Eq. (1.2). The term $H(p, Q)$ is a constant for $p=p_{s}, p_{0}$ and it satisfies

$$
\begin{aligned}
H(p, Q) & \leqslant \int \exp \left[\left(\alpha-\mathbf{x}^{2} / 2\right) \mathbf{x}^{2} / F\right] d \mathbf{x}-1 \\
d H(p, Q) / d t & =\frac{1}{2} F \int p(\nabla \ln p / Q)^{2} d \mathbf{x} \geqslant 0
\end{aligned}
$$

3. Minor corrections are: In Eq. (2.6a) replace $\theta$ by $|\theta|$. In Eq. (2.28) replace $\psi_{1}$ by $\phi$.

\footnotetext{
${ }^{1}$ This paper originally appeared in J. Stat. Phys. 48:571-591 (1987).

${ }^{2}$ Departamento de Física Teórica, Universidad de Sevilla, 41080 Sevilla, Spain.
} 
References 2 and 3 also deal with mean-field models having stable time-periodic probability densities. Shiino ${ }^{(2)}$ also studies our Eq. (1.3), while Schentzow ${ }^{(3)}$ analyzes the case of a mean-field Brusselator with additive white noise. We are grateful to Prof. D. Dawson for bringing these works to our attention.

\section{REFERENCES}

1. M. Shiino, Phys. Lett. 112A:302 (1985).

2. M. Shiino, Phys. Lett. 111A:396 (1985).

3. M. Schentzow, Prob. Theory Rel. Fields 72:425 (1986). 